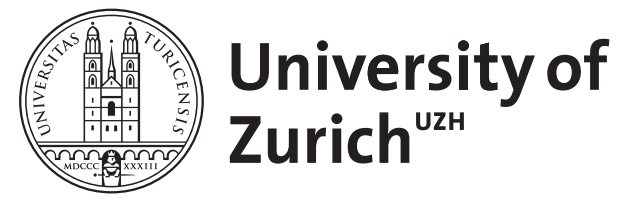

Zurich Open Repository and Archive

University of Zurich

University Library

Strickhofstrasse 39

CH-8057 Zurich

www.zora.uzh.ch

Year: 2013

Blinder Einsatz von Statinen könnte den Augen schaden

Djalali, Sima

DOI: https://doi.org/10.1024/1661-8157/a001499

Posted at the Zurich Open Repository and Archive, University of Zurich

ZORA URL: https://doi.org/10.5167/uzh-90671

Journal Article

Accepted Version

Originally published at:

Djalali, Sima (2013). Blinder Einsatz von Statinen könnte den Augen schaden. Praxis, 102(25):1555-1556.

DOI: https://doi.org/10.1024/1661-8157/a001499 


\section{Blinder Einsatz von Statinen könnte den Augen schaden}

\section{Frage:}

Erhöhen Statine das Kataraktrisiko?

\section{Hintergrund:}

Dass Statine nicht nur auf den Cholesterinspiegel wirken, ist immer wieder Gegenstand hoffnungsvoller Forschungshypothesen. Hinweise, dass Statine den Alterungsprozess der Augenlinse und damit die Eintrübung und Kataraktentwicklung beeinflussen könnten, existieren bereits seit Ende der Neunzigerjahre. Allerdings ist bislang nicht klar, ob die Statine das Kataraktrisiko senken oder erhöhen - Studienergebnisse existieren für beide Hypothesen. Nun soll eine retrospektive Analyse der umfassenden Patientendaten einer amerikanischen Gesundheitsversorgungsorganisation Aufschluss geben.

\section{Einschlusskriterien:}

- Registrierte Patienten des militärischen Gesundheitsversogungsnetzwerks San Antonio

- 30-85 Jahre alt

- Mindestens eine ambulante Arztkonsultation zwischen Oktober 2003 und September 2005 (Baselineperiode) und eine weitere zwischen Oktober 2005 und März 2010 (Follow-upPeriode)

\section{Ausschlusskriterien:}

- Statintherapie für weniger als 90 Tage

- Beginn der Statintherapie nach September 2005

- Traumapatienten

\section{Studiendesign und Methode:}

Retrospektive Kohortenstudie auf Basis von medizinischen und administrativen Routinedaten der Leistungserbringer und -träger im Gesundheitsversorgungsnetzwerk. Patienten mit Statingebrauch wurden über Rezeptierungsdaten identifiziert, die Kataraktdiagnose über ICD9-Codierungen festgestellt. Die unterschiedlichen Statinpräparate und -dosen, die die Patienten erhalten hatten, wurden in Simvastatinäquivalente umgerechnet und die kumulative Simvastatindosis (Simvastatinjahre) erhoben.

\section{Studienort:}

San Antonio, Texas, USA

Interventionen:

- Statintherapie über mindestens 90 Tage

\section{Outcome:}

Primärer Outcome

- Risiko einer Kataraktentwicklung unter Statintherapie

\section{Resultat:}

- $13^{\prime} 626$ Patienten unter Statintherapie und 32'623 Patienten ohne diese Medikation wurden aus den Patientenakten identifiziert. Um die Vergleichbarkeit zu gewährleisten und den Einfluss von Komorbiditäten, anderen Medikationen und weiterer Störfaktoren statistisch zu eliminieren, wurden die Teilnehmer gematcht und zwei vergleichbare 
Gruppen à 6972 Patienten gebildet. Die mittlere Therapiedauer lag bei 1593 Tagen, die mittlere kumulative Dosis bei 141,8 Simvastatinjahren.

- Das Kataraktrisiko unter Statintherapie war signifikant erhöht (Odds ratio 1,09;95\% Cl 1,02-1,17) und war abhängig von der Dauer und der kumulativen Dosis (Simvastatinjahre), nicht aber von der Höhe der verordneten Tagesdosis.

- Vor allem das Risiko für präsenile, senile und traumatisch bedingte Kataraktformen war signifikant höher bei Patienten mit Statineinnahme. Das Risiko für andere Augenerkrankungen, systemische Krankheiten oder iatrogen hervorgerufene Kataraktformen war nicht erhöht.

- Verschiedene statistische Analysen zeigten konsistent Statine als unabhängigen Risikofaktor für die Kataraktentwicklung.

\title{
Kommentar:
}

- Die Studie wählt eine Forschungsmethode, die durch elektronische Informationssysteme ermöglicht wird: die retrospektive Analyse von medizinischen und administrativen Routinedaten. Während die Daten bei klassischen Kohortenstudien nur punktuell im Rahmen von Follow-up-Terminen erhoben werden, ermöglicht die Extraktion von Routinedaten aus den Akten der Gesundheitsversorger eine annähernd lückenlose Dokumentation von Patientenverläufen. In dieser Studie wurde der Datenpool eines Gesundheitsversorgungsnetzwerks verwendet, in dem sogar Daten von externen Leistungserbringern und Apotheken integriert sind. Die Datengrundlage der Studie ist damit als robust anzusehen.

- Die Kataraktentstehung ist multifaktoriell und Patienten, die Statine erhalten, sind oft multimorbid. Für alle bekannten Einflüsse wurde in der vorliegenden Studie mit statistischen Verfahren korrigiert. Bei kleinen Stichproben kann die Anwendung solcher statistischer Verfahren problematisch sein und zu Verzerrungen der Ergebnisse führen. Die Stichprobe in der vorliegenden Studie war mit über 45`000 Patienten jedoch gross genug, so dass die Analyseergebnisse als robust angesehen werden können.

- Fazit: Das Risiko der Kataraktentwicklung sollte bei Beginn einer Statintherapie in der Entscheidungsfindung miteinbezogen und gegen die Indikation aufgewogen werden, insbesondere beim primärpräventiven Einsatz.

\section{Literatur:}

Leuschen J, Mortensen EM, et al.: Association of Statin Use With Cataracts: A Propensity Score-Matched Analysis. JAMA Ophthalmol 2013; doi: 10.1001/jamaophthalmol.2013.4575. [Epub ahead of print]

\author{
Verfasser: \\ Dr. med. Sima Djalali \\ Institut für Hausarztmedizin \\ Universität Zürich \\ Pestalozzistrasse 24 \\ 8091 Zürich \\ sima.djalali@usz.ch
}

